\title{
Integrating tuberculosis case finding and treatment into focused antenatal care in Kenya
}

Charlotte E. Warren

Population Council

Annie Mwangi

Population Council

Follow this and additional works at: https://knowledgecommons.popcouncil.org/departments_sbsr-rh

Part of the International Public Health Commons, Maternal and Child Health Commons, and the Social and Behavioral Sciences Commons

How does access to this work benefit you? Let us know!

\section{Recommended Citation}

Warren, Charlotte E. and Annie Mwangi. 2008. "Integrating tuberculosis case finding and treatment into focused antenatal care in Kenya," FRONTIERS Final Report. Washington, DC: Population Council. 


\title{
Integrating Tuberculosis Case Finding and Treatment into Focused Antenatal Care in Kenya
}

\author{
Charlotte Warren and Annie Mwangi
}

August 2008

This study was made possible by the generous support of the American people through the United States Agency for International Development (USAID) under the terms of Cooperative Agreement Number HRN-A-00-98-00012-00, FRONTIERS, and Subproject Number 8816 53098. The contents are the responsibility of the Population Council and do not necessarily reflect the views of USAID or the United States Government.
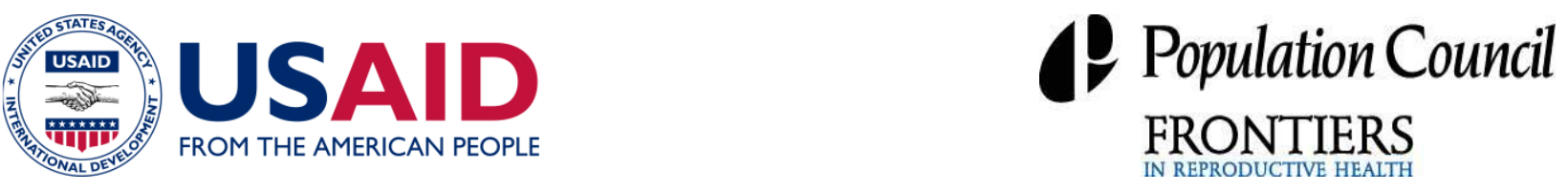
(C) 2008 The Population Council, Inc.

Suggested citation: Warren, Charlotte and Annie Mwangi. 2008. "Integrating tuberculosis case finding and treatment into focused antenatal care in Kenya," FRONTIERS Final Report. Washington, DC: Population Council.

Any part of this publication may be reproduced without permission for limited distribution provided it is distributed without charge and the Population Council is acknowledged as the source. The Population Council would appreciate receiving a copy of any materials in which the text is used. 


\section{SUMMARY}

Tuberculosis (TB) remains a major public health problem in Kenya, with an incidence of about 619 per 100,000 population, or roughly 200,000 new cases per year (WHO, 2006). The burden of disease for tuberculosis in Kenya accounts for over 145,000 disability-adjusted life years annually, with the health consequences being worse for females in the reproductive age group (MOH 2005). An estimated 19 percent of maternal deaths are attributable to indirect obstetric causes, the majority of which are caused by malaria, anemia, and HIV/AIDS, and HIV/AIDSrelated deaths include those due to TB (WHO 2003). Any attempt to improve maternal health must also include careful screening and investigation for TB in pregnant women in areas of high HIV and TB prevalence (Khan et al 2001).

In Kenya most pregnant women (90\%) attend antenatal care at least once and $52 \%$ make four or more visits (KDHS 2003). Detection of HIV and TB are recommended as part of the standard Focused ANC (FANC) package, but while efforts to increase HIV detection have accelerated recently through the introduction of PMTCT procedures, little effort has been made to increase detection of TB. The management of TB in pregnancy and during the puerperium period is also not integrated within maternal and child health care programs. This lack of integration of TB and PMTCT activities in FANC implies that mothers who are HIV positive and are seeking care are likely to get help only for services that target HIV/AIDS and pregnancy, and miss out altogether on those services that target TB patients. It is not know how many infants are born to women with TB in Kenya.

The Population Council/FRONTIERS program, in collaboration with the National Leprosy and Tuberculosis Programme (NLTP), the Division of Reproductive Health (DRH) and the Provincial Health Management Team (PHMT) in Western Province (WP), received PEPFAR funding to improve screening for TB for pregnant women in general including HIV positive pregnant women, in Kenya. An intervention was tested to strengthen TB case detection as part of the FANC and PMTCT program. The aim was to ensure that $\mathrm{MCH}$ providers, in addition to providing ANC/PMTCT services, also screen and assess the client's need for TB services and refer for further management.

To facilitate implementation of the intervention, a situation analysis of TB case finding and treatment in FANC was conducted in October 2005 in six hospitals in Western Province. An evaluation of the intervention was then carried out in October 2007. FRONTIERS and the MOH developed a module for screening for TB in pregnancy and incorporated it into the $4^{\text {th }}$ Edition of the FANC Orientation Package (MOH/JHPIEGO 2007). A job aid was also produced as a checklist for providers to ask questions relating to signs and symptoms of TB. In total, 83 health care providers from Western province attended a three-day training session on FANC- TB. Teams from facilities included staff from the MCH-FP units, TB units and laboratory staff who were trained together. Subsequently the facility teams developed action plans for screening for TB and improving linkages between the departments within the facilities. These action plans were also used during supervision visits. 
Facilities were either already prepared to offer TB screening or needed only minimal adjustments at baseline to provide TB screening in FANC. Western Province hospitals had over four-fifths of the necessary infrastructure, equipment, drugs, guidelines and supplies. The model is therefore quite feasible in this setting, but there were some actions in terms of strengthening operational policies that are required for successful scale-up. One key element was to bring providers from the ANC unit, TB unit and laboratory together from each facility for team training as one group. This provided a foundation to improve referrals and a consistent approach to meeting the needs of pregnant women who have TB.

Overall the quality of care in screening for TB has certainly improved. The most significant increases were noted during the first ANC visit. At baseline, less than one-tenth of providers were observed asking about any persistent cough in any of the antenatal visits. This improved to two-thirds in the first visit at the endline. Screening for other areas was less likely to be done in subsequent visits but significant improvements were also noted.

Districts that wish to increase screening for TB at lower level facilities, such as health centers and dispensaries, must review the referral mechanisms for ensuring that pregnant women suspected of having TB do not have to travel far. The provision of sputum pots at the community level that could then be transported by Public Health Technicians (PHTs) on certain days would reduce the burden for pregnant women. The acceptability to providers of TB screening in ANC is demonstrated through the widespread use of the new orientation package as well as ease in which the providers have taken it on board within the FANC services. Screening for TB has become part of routine activity for a majority of nurses.

Effectiveness of integrating TB into FANC is demonstrated by the increased number of pregnant women who were detected to have TB and were started on treatment. Although it is estimated that half of all those with HIV are also infected with TB, the women attending antenatal care appear to be a reasonably 'healthy' population. Nevertheless, the simplicity of this approach means that women can be screened for TB in the antenatal setting as well as having the process replicated in postnatal or other RH environments. This may be a more effective intervention in high TB and HIV prevalence areas. In developing the tools for the study (i.e., TB module and job aids), program leaders from the NLTP and DRH were brought together, probably for the first time, to address the needs of women who may have TB. As far as NLTP is concerned, every case of TB detected is a bonus given their strategic objective in increasing case detection.

\section{Recommendations for key stakeholders emerging from this effort include:}

- Hold further consultations with key actors to ensure institutionalization and standardization of TB screening in FANC. This includes the integration or linkages with PMTCT- HIV and STI and postnatal services, and incorporating or updating curricula of pre-service training institutions and professional bodies on TB case management guidelines.

- Scale up TB screening in other service areas such as postnatal care through the training of providers with a focus on providing a comprehensive package of care for the mother and her infant. In addition, scale up screening of TB should be extended to the provinces with the highest $\mathrm{TB}$ rates. 


\section{Scaling up activities}

In early 2008 the $\mathrm{MOH}$ replicated the introduction of screening for TB in ANC in a poor urban environment. Pumwani Maternity Hospital is one of the largest maternities in sub-Saharan Africa with many of its clients living in informal crowded settlements in Nairobi. The facility readiness score for Pumwani (38 out of 52, or 73\%) was less than that of the Western Province hospitals, mainly because there is no TB unit and therefore no TB tests, drugs or copies of guidelines. Provider knowledge was similar to that of the hospitals in Western Province, although the Pumwani providers had less knowledge on TB. All clients who are suspected to have TB are referred outside of the facility. Plans include making the TB test and drugs available on site so that pregnant women with TB can receive comprehensive care in one place.

Service providers from Pumwani Maternity Hospital (78) and private and public feeder clinics (31) were oriented in FANC/TB in five separate training sessions between February and April 2008. Facilitators included trainers from Population Council/FRONTIERS, the Pumwani Midwifery School and the Provincial Nursing Office. An evaluation of the impact of the training and supervision will be carried out later in the year through a separate funding mechanism.

A baseline assessment was also carried out in early 2008 in the remaining district hospitals in Western Province. This information is being made available for APHIA II Western which will take the lead in strengthening screening for TB in ANC in those facilities and across the province. 


\section{ACKNOWLEDGMENTS}

We wish to acknowledge the support from the Ministry of Health, National Program for Leprosy and Tuberculosis and the Division of Reproductive Health. Special thanks go to the Provincial Health Management Team, Western Province, the District Health Management Teams of Bungoma, Kakamega, Mumias, Busia and Teso and the six hospitals; Provincial General Hospital, Kakamega, Webuye Hospital, Bungoma District Hospital, Teso District Hospital, Busia District Hospital and St Mary's Hospital, Mumias.

Special thanks go to the data management unit, research assistants and all the women who participated in the study; without them the research would not have been possible. 


\section{ACRONYMS AND ABBREVIATIONS}

$\begin{array}{ll}\text { AIDS } & \text { Acquired Immuno Deficiency Syndrome } \\ \text { ANC } & \text { Antenatal Clinic } \\ \text { ARV } & \text { Anti retro viral } \\ \text { BCG } & \text { Bacille Calmette Guerin } \\ \text { CS } & \text { Child Survival } \\ \text { DHMT } & \text { District Health Management Team } \\ \text { DTLC } & \text { District TB and Leprosy Officer } \\ \text { DOTS } & \text { Directly Observed Treatment Short course } \\ \text { DRH } & \text { Division of Reproductive Health } \\ \text { FANC } & \text { Focused Antenatal Care } \\ \text { FP } & \text { Family Planning } \\ \text { HIV } & \text { Human Immuno-deficiency Virus } \\ \text { KSPA } & \text { Kenya Service Provision Assessment } \\ \text { MCH } & \text { Maternal and Child Health } \\ \text { MOH } & \text { Ministry of Health } \\ \text { NASCOP } & \text { National AIDS and STDs Control Program } \\ \text { NLTP } & \text { National Leprosy and Tuberculosis Programme } \\ \text { PMCT } & \text { Prevention of Mother to Child Transmission } \\ \text { PMH } & \text { Pumwani Maternity Hospital } \\ \text { PNC } & \text { Postnatal care } \\ \text { PTB } & \text { Pulmonary Tuberculosis } \\ \text { RH } & \text { Reproductive Health } \\ \text { STI } & \text { Sexually Transmitted Infections } \\ \text { TB } & \text { Tuberculosis } \\ \text { WHO } & \text { World Health Organization }\end{array}$




\section{BACKGROUND}

Tuberculosis remains a major public health problem in Kenya, with an incidence of about 619 per 100,000 population, or roughly 200,000 new cases per year (WHO, 2006). The burden of disease for tuberculosis in Kenya accounts for over 145,000 disability-adjusted life years annually, with the health consequences being worse for females in the reproductive age group (MOH 2005). Tuberculosis kills more adults than malaria and is one of the leading causes of mortality among women of reproductive age worldwide. According to WHO, Kenya is among the 22 countries worst hit by the TB epidemic worldwide. Tuberculosis in Kenya accounts for over 145,000 disability adjusted life years (DALYs) with the situation being worse for females in the reproductive age group (WHO, 2003). The incidence of TB during pregnancy in Kenya is not known; however, an estimated 19 percent of maternal deaths are attributable to indirect obstetric causes, the majority of which are caused by malaria, anemia, and HIV/AIDS, where HIV/AIDS related deaths also includes TB as a cause of death.

According to Pillay et al (2004), "tuberculosis is the commonest HIV-1-related disease and the most frequent cause of mortality in young women in endemic regions". Tuberculosis and HIV-1 are independent risk factors for maternal mortality and adverse perinatal outcomes, and in combination have a greater impact on these parameters than their individual effects. In referral health centers in southern Africa, around one-sixth of all maternal deaths are due to tuberculosis/HIV-1 co-infection. One-third (37\%) of HIV-1-infected mothers with tuberculosis are severely immuno-compromised, with CD4 counts of fewer than 200 cells/microL, compared with less than 20 percent in mothers recruited into major mother-to-child intervention trials in Europe. Babies born to mothers with tuberculosis/HIV-1 co-infection also have higher rates of prematurity, low birth weight, and intrauterine growth restriction. Transmission rates of HIV-1 from mother to infant are around 25-45 percent in resource-limited settings, while that for mother-to-child-transmission of tuberculosis is 15 percent within 3 weeks of birth. Any attempt to improve maternal health must also include careful screening and investigation for tuberculosis in high-risk pregnant women (Khan et al 2001).

In order to address challenges posed by TB, the Ministry of Health (MOH) of Kenya, through the National Leprosy and Tuberculosis Program (NLTP), has identified early case detection and treatment of the detected cases as a key strategy in TB control. The aim of case finding is to diagnose and treat TB cases as early as possible, mainly through passive case finding, timely treatment and contact tracing. The NLTP strategy also encourages the decentralization of diagnostic or treatment centers to increase access to TB services, TB/HIV collaboration, better coordination with the private health sector and increased awareness of TB control among health workers and the communities. While the strategy underlines TB/HIV collaboration, actual implementation of this strategy has been limited in scope. The strategy fails to highlight linkages between TB, PMCT and above all focused ANC (FANC); yet focused ANC is now considered a critical entry point for a variety of services to pregnant women.

In Kenya most pregnant women (90\%) attend antenatal at least once and 52\% make four or more visits (KDHS 2003). To promote the health and survival of mothers and babies, Kenya's $\mathrm{MOH}$ has adapted the WHO focused ANC (FANC) package that includes a minimum of four targeted visits for ANC. The focused ANC guidelines emphasize comprehensiveness and that each visit 
should have a clearly defined purpose. Detection of HIV and TB are recommended as part of the standard FANC package. Although efforts to increase HIV detection have accelerated recently through the introduction of PMTCT procedures, little effort has been made to increase detection of TB, either among HIV+ pregnant women or among pregnant women generally. The management of TB in pregnancy and during the puerperium period is currently not integrated under the maternal and child health care programs. This lack of integration of TB and PMTCT activities in FANC implies that mothers who are HIV positive and are seeking care are likely to get help only for services that target HIV/AIDS and pregnancy, and miss out altogether on those services that target TB patients.

Existing guidelines are weak and/or not clear on TB detection in the reproductive health setting. For example, both PMTCT and FANC guidelines do not include a TB screening checklist. The maternal health card only requires the provider to take medical/family history on TB. The National TB guidelines are also not clear on the management of TB in pregnant women. The guidelines for ARV mention that clients should not take ARV and first phase TB drugs at the same time. The National Guidelines for PMTCT encourage health care providers working in antenatal clinics to provide a comprehensive package of services, to include counseling and testing for HIV as well as information on the interaction between HIV and TB during group/individual counseling sessions with pregnant women. The national PMTCT guidelines recognize that TB is a common opportunistic infection in HIV-positive pregnant women and recommend that FANC providers should give a full physical examination to all pregnant women identified to be HIV positive, focusing on HIV-related symptoms and illnesses and signs of opportunistic infection especially TB.

Although TB detection is encouraged among clients with HIV in the FANC clinics, providers in these settings are not trained in diagnosing and managing TB cases, neither are TB providers familiar with managing TB in pregnancy. The 1999 Kenya Service Provision Assessment (KSPA) notes that more than half of the health workers providing STI/HIV/AIDS had not received training in management of TB cases.

\section{Proposed solution}

To address these problems, the Population Council's FRONTIERS program proposed to test the introduction of an intervention that will strengthen $T B$ case detection as part of the focused ANC and PMTCT program. The aim was to ensure that MCH providers, in addition to providing ANC/PMTCT services, can also screen and assess the client's need for TB services and refer cases for clinical management.

Traditionally, TB programs have been run as parallel activities within the MOH. Since integration is a new approach it is important that the feasibility of combining these activities is tested. In phase one of the study we assessed the feasibility of introducing a checklist for TB case finding and subsequent treatment among pregnant women in Kenya, and documented the effect of this intervention on the proportion of pregnant women that are detected to have TB and are correctly managed. The study sought to address the following questions: 
- What are the obstacles to successful integration of TB case finding in focused ANC and PMTCT?

- Will ANC and TB providers be willing to introduce TB case detection in the ANC clinic?

- Will the integration of TB case finding in PMTCT and FANC improve TB case finding in pregnant women generally and particularly for TB in HIV+ women.

- Will the intervention be feasible and acceptable in the public health service?

\section{Objectives}

\section{Overall}

The overall objective of the study was to improve access to care and treatment for TB for pregnant women in general and HIV positive pregnant women in Kenya.

\section{Specific Objectives}

- Improve procedures and protocols for TB, PMTCT and focused ANC through strengthening linkages that improve client flow;

- Improve knowledge about TB among health workers providing TB, PMTCT and ANC services, and strengthen their capacity to detect TB in pregnant women, and to refer and/or manage suspected cases;

- Improve the referral mechanism for managing and treating HIV+ pregnant women and pregnant women in general suspected of having TB infection;

- Document the feasibility of integrating screening and detection of TB into PMTCT/focused ANC;

- Evaluate the effect of integrating TB screening, case detection and treatment into focused ANC and PMTCT on the proportion of TB-positive women treated. 


\section{METHODOLOGY}

This evaluation was designed on the basis that no systematic effort to detect TB exists during ANC services, and that TB case finding was not an integral component of FANC and PMTCT. Consequently, the study used a post-intervention design to test the study hypothesis (see diagram below). The hypothesis will be accepted if the descriptive measurements for the different variables are significantly greater than zero and the package has the potential for a public health impact in the study sites. The experimental group of six facilities where the intervention (X) was introduced was observed six months after its introduction, when measures of the key dependent variables were collected $\left(0_{1}\right)$.

These six facilities were selected from a list of hospitals in Western Province with the following criteria: availability of FANC, PMTCT, functioning laboratory and TB unit; and no other organization is providing intensive support to PMTCT to ensure that possible confounding factors are controlled for.

Study sites

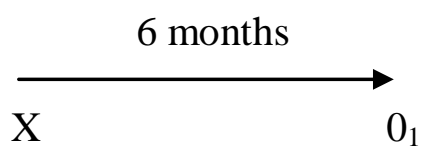

Where:

X: A set of four complementary interventions introduced:

- Clinic readiness to offer integrated TB/PMTCT and FANC package

- Development of training materials

- Improving provider knowledge

- Proactive screening of pregnant women for TB symptoms and referral.

01 Post intervention measurements of:

- Number of clients attending PMTCT, ANC and TB services and services received

- Quality of TB screening in HIV+ pregnant women and pregnant women in general

- Referral for TB test and treatment

- Provider competence

- Level of TB/PMTCT/ANC integration.

\section{Hypothesis}

Introduction of TB screening in focused ANC will significantly increase the proportion of pregnant women screened for TB, the proportion of $\mathrm{HIV}+$ pregnant women screened, and the number of women being treated jointly for active TB and HIV. 


\section{Operational definitions of key variables}

Screening for $\mathrm{TB}$

- Proportion of pregnant women screened for TB in ANC /PMTCT setting.

\section{Case detection and treatment}

- Proportion of pregnant women suspected to have TB that have a first sputum test.

- Proportion of pregnant women suspected to have TB and referred for subsequent test that are actually tested.

- Proportion of pregnant women diagnosed with Active TB referred for TB treatment.

\section{Provider Knowledge, Competence and Behavior}

- Proportion of TB providers knowledgeable of TB in pregnancy.

- Proportion of ANC/PMCT providers who, in addition to providing ANC/PMCT services, are competent to screen and assess client's need for TB services and refer.

\section{Improved linkages between TB, ANC/PMCT}

- Number of clients receiving PMCT, ANC and TB services referred to and from these services.

- Number of clinics offering related services on the same day

\section{Study sites}

Six hospital antenatal clinics were selected purposively in Western Province that fulfilled the criteria mentioned above: Kakamega Provincial General Hospital; Bungoma, Busia and Teso district hospitals; Webuye Sub-District Hospital; and St Mary's Mission Hospital, Mumias.

\section{Facility Assessment - documenting feasibility and acceptability of the intervention:}

Indicators for measuring facility readiness to offer the strengthened services were measured before the intervention was introduced in all four facilities through an inventory of the resources necessary and available in each facility, e.g. infrastructure, equipment, commodities, test kits, stationary client cards and notes, TB and ARV drugs and other medications required to provide the services as well as staffing. The intervention did not involve strengthening the infrastructure or increasing the staff of the clinics and therefore these items were not measured after the intervention. While it is recognized that the coverage measures are sensitive to changes in staff and attrition and transfers would be present in any facility over the comparable study, so the study was done under the conditions required for eventual scale up. Changes in service provision and referral were tracked through client registers. Clinics maintained client registers that indicate the number of clients, types of services received and referrals.

Provider interviews: Short structured interviews were carried out among PMCT/ANC and TB providers to assess their knowledge of manifestations of TB in pregnancy, comprehensive ANC, and screening and detection of TB in pregnancy immediately before the training. The interviews 
targeted all service providers to be trained during the intervention. The gaps in knowledge were addressed during the development of the TB training materials.

Structured observations of client-provider interactions were used to measure provider competence and their ability to follow service provider guidelines for care. Using a standardized checklist, qualified nurse-midwives were trained to observe and record the quality of care provided at four points in time during the antenatal period: first visit, second visit, third visit and fourth visit. All observations were carried out during the same time period with cross-sectional samples of women for each category. All women attending the clinic for these services during the data collection period were approached and requested for permission to observe their consultation. Mean scores were computed for sets of each indicator and then aggregated across all indicators to give the composite score for quality of antenatal care given. This method was used to demonstrate overall improvements in care rather than individual outcomes.

Table 1: Data collection tools and sample sizes for quality of care

\begin{tabular}{l|cc}
\hline & Baseline & Endline \\
Tools and sample sizes for quality of care & 2005 & 2007 \\
\hline Provider interview: training audit & 45 & - \\
Observed Client-provider interaction first ANC visit & 69 & 36 \\
Observed Client-provider interaction second ANC visit & 56 & 36 \\
Observed Client-provider interaction third ANC visit & 53 & 36 \\
Observed Client-provider interaction fourth ANC visit & 6 & 25 \\
Facility assessment and service statistics & 6 \\
\hline
\end{tabular}




\section{THE INTERVENTION}

A set of four complementary interventions were introduced:

1. Clinic readiness to offer integrated TB/PMTCT and FANC package

2. Development of TB training materials

3. Provider orientation

4. Screening of pregnant women for TB symptoms and referral

\section{Clinic readiness to offer integrated TB/PMTCT and FANC package}

Clinics found to lack adequate capacity to offer the combined package of PMTCT, TB and focused ANC (FANC) were strengthened. Support from the NLTP, the DRH, and the District Health Management Team (DHMT) ensured that the clinics had sufficient supplies, equipment, and revised guidelines to offer the services. Results from the facility assessment tool outlined gaps that were missing. Registers and drugs were made available through the DHMT. The orientation materials on TB in FANC and job aids were printed and distributed by the Population Council through the DHMTs.

\section{Development of training materials for provider orientation}

Prior to this study there were no training materials for offering TB screening in reproductive health settings. A task force was constituted at the beginning of the project period and included members of the NLTP Unit and DRH as well as representatives from the study districts. MOH documents guided this review, including: NASCOP Strategic Plan (2005); National Guidelines for Prevention of Mother to Child Transmission of HIV/AIDS (2005); National Leprosy and Tuberculosis Program Strategic Plan (2003); Guidelines for Tuberculosis and Leprosy Control (2004); Guidelines to Antiretroviral Therapy in Kenya (2005); and the Orientation Package for Focused Antenatal Care and Malaria in Pregnancy (2002).

Existing service registers, including the TB patient record card, TB appointment card, TB treatment register, TB ambulatory treatment register, PMCT register, antenatal register, and laboratory registers, were reviewed. Providers of each type of service were consulted and involved in designing the best way of ensuring integration of the TB/PMTCT/FANC services.

The project task force, jointly led by the MOH and FRONTIERS, developed a module for TB in pregnancy. The TB module includes both factual technical updates such as diagnosis/detection, counseling and treatment, as well as information about what services should be provided (such as sputum examination and free treatment for TB). There is also a screening component focusing on TB detection. This module was incorporated into the National Focused ANC Orientation Package ( $\mathrm{MOH} 4^{\text {th }}$ Edition 2007), which is used to update all service providers in focused ANC. The task force also developed a job aid for screening all women attending focused antenatal care (and PMTCT) which serves as a checklist for providers in the clinic (see appendix).

\section{Provider orientation in TB and ANC}

Participants for the 3-day training were selected from the six hospitals. A schedule developed for the TB/ANC orientation package guided the training (see appendix). The first three-day workshop was conducted in Bungoma Town in May 2006 for health providers from the six sites. 
Fifty-nine health care providers were trained from the six study sites in two groups. A further 24 participants were updated in May 2007 due to staff attrition and transfers out of the district. The course equipped service providers with knowledge and skills to be able to screen and refer ANC clients appropriately and to manage TB when suspected. In addition it was expected to improve providers' attitudes towards case finding of TB and comprehensive management of individual women attending antenatal care.

Participants were grouped in "Facility Teams" to share information and discuss joint referral guidelines and checklists within their hospitals to improve quality of care for clients moving between departments. In addition to developing a facility action plan, the trained health providers were asked to carry out an "awareness day" for their colleagues, reaching an estimated 1,600 health workers by the end of the second year within their facilities and districts to create an understanding of TB and HIV co-infection in pregnancy and the importance of integrating TB services within PMTCT activities.

The participants included staff from the MCH/FP clinics as well as staff from ANC-PMTCT, $\mathrm{TB} / \mathrm{Chest}$ clinic and the laboratories. Managers with supervisory roles for all aspects of care and support were also trained, such as the district public health nurse, public health officers, district TB coordinator and district medical lab technologist.

Participants took part in knowledge tests before and after the training. The questions mainly revolved around components of focused antenatal care (ANC, malaria in pregnancy, PMTCT and TB). Among providers in group one in Western Province, the pre-post scores increased significantly from a median of 60 percent (range: $35 \%-85 \%$ ) prior to training to a median of 76 percent (range: 58\% - 92\%) afterwards. Group two also increased significantly from a median of 56 percent (range: $41 \%-78 \%$ ) to a median of 74 percent (range: $58-94 \%$ ). The providers scoring at the lower end of the range were mainly laboratory technologists $(n=13)$ and are not expected to provide clinical care. In-service training would be required to ensure that all staff has the skills to do the expected tasks in each facility.

\section{Screening of pregnant women for TB symptoms and referral}

All women attending ANC were to be screened for TB using the checklist (see appendix). All women were asked if they have had a cough lasting more than two weeks and whether she had any enlarged glands. If either of the answers are 'yes' the rest of the questions are asked (i.e. prolonged cough, night sweats and weight loss). If the provider suspected TB infection the client was counseled and given a sputum pot to provide a sputum specimen and referred to the laboratory. The nurse filled out the laboratory request form to give to the client to take with the specimen. A notation is usually made on the ANC card and in the ANC register. The health care provider is expected to explain the importance of testing three specimens. The client was advised that three specimens make a firm diagnosis of TB. 


\section{MONITORING}

Services data from the TB, ANC and laboratory registers was collected from the facilities by staff in-charge of the facilities and sent to the Population Council in Nairobi.

\section{TB screening}

The number of clients screened for TB in ANC increased overall by 43 percent during the months of May to October between 2006 and 2007, although there were substantial variations between hospitals. It is expected that all pregnant women attending ANC are screened for TB. The number of pregnant women who were referred from ANC to the laboratory for a sputum test decreased from 55 in 2006 to 13 in 2007 . Table 2 illustrates that very few of the pregnant women screened were detected with TB.

Table 2: TB screening assessment data - May to October 2006 and 2007

\begin{tabular}{l|cccccc}
\hline \multirow{2}{*}{ Facility } & \multicolumn{2}{c}{$\begin{array}{c}\text { Screened for } \text { TB } \\
\text { in }\end{array}$} & $\begin{array}{c}\text { Pregnant women's } \\
\text { sputum }\end{array}$ & $\begin{array}{c}\text { Pregnant women } \\
\text { with } \text { TB }\end{array}$ \\
\cline { 2 - 6 } Bungoma DH & $\mathbf{2 0 0 6}$ & $\mathbf{2 0 0 7}$ & $\mathbf{2 0 0 6}$ & $\mathbf{2 0 0 7}$ & $\mathbf{2 0 0 6}$ & $\mathbf{2 0 0 7}$ \\
Busia DH & 2503 & 2087 & 2 & 0 & 1 & 0 \\
Teso DH & 1061 & 1741 & 5 & 2 & 0 & 2 \\
PGH Kakamega & 1024 & 1004 & 10 & 3 & 1 & 0 \\
St Mary's Hospital & 1103 & 6621 & 28 & 8 & 1 & 0 \\
Webuye SDH & 1840 & 3586 & 8 & 0 & 1 & 0 \\
Aggregate & 462 & 1704 & 2 & 0 & 0 & 0 \\
\hline
\end{tabular}

\section{Case detection and treatment}

The TB case notification rate (CNR) for Kenya in 2006 was 325 per 100,000 of the population. The estimated population for Western Province is around 4 million. Therefore the expected number of TB cases would be 13,000. The HIV prevalence rate from surveillance sites among pregnant women is $7.4 \%$. Given that the number of pregnancies account for approximately four percent of the total population in any given year or time of year in which measurement took place (such as May-October), Western Province could expect around 160,000 pregnancies during this period. If the HIV prevalence is $7.4 \%$, then the potential number of HIV positive pregnant women would be 11,840 .

Therefore the expected number of TB cases among HIV positive pregnant women in Western Province (325 x 11,840/100,000) would equal 39, given the prevailing CNR. However it is likely that this would be higher as HIV positive people have approximately $50 \%$ chance to develop TB (Kenya TB and Leprosy Treatment Guidelines 2003). It would be expected, therefore, that many more pregnant women than recorded have TB.

Table 3 presents the number of new cases of TB registered in the six hospitals. It is not known whether the female clients were pregnant or not. The figures are highly variable by size and type of facility. In most sites, however, the proportion of female cases of TB detected improved over the study period. 
Table 3: TB monitoring data for 6 month periods May-Oct 2006 and May-Oct 2007

\begin{tabular}{lcccccccc}
\hline & $\begin{array}{c}\text { New TB } \\
\text { cases }\end{array}$ & $\begin{array}{c}\text { New female } \\
\text { clients with } \\
\text { TB }\end{array}$ & $\begin{array}{c}\text { Female } \\
\text { proportion of } \\
\text { new cases (\%) }\end{array}$ & $\begin{array}{c}\text { New clients with } \\
\text { Pulmonary TB }\end{array}$ \\
Hospital & $\mathbf{2 0 0 6}$ & $\mathbf{2 0 0 7}$ & $\mathbf{2 0 0 6}$ & $\mathbf{2 0 0 7}$ & $\mathbf{2 0 0 6}$ & $\mathbf{2 0 0 7}$ & $\mathbf{2 0 0 6}$ & $\mathbf{2 0 0 7}$ \\
& & & & & & & & \\
Bungoma DH & 270 & 185 & 66 & 93 & 25 & 50 & 88 & 108 \\
Busia DH & 177 & 204 & 70 & 103 & 6 & 51 & 141 & 204 \\
Teso DH & 249 & 90 & 6 & 37 & 2 & 41 & 17 & 77 \\
PGH Kakamega & 700 & 460 & 352 & 219 & 50 & 48 & 569 & 430 \\
St Mary's Hospital & 32 & 82 & 8 & 32 & 25 & 39 & 15 & 61 \\
Webuye SDH & 161 & 178 & 83 & 95 & 52 & 53 & 121 & 148 \\
\hline
\end{tabular}

\section{FINDINGS}

This section describes baseline results obtained from the facility assessment, provider interviews and provider-client interactions in the antenatal clinics in six hospitals in Western Province.

\section{Facility readiness}

Health facilities have the capacity to integrate TB screening into FANC

At each hospital we assessed the availability of essential items to provide Focused ANC and TB screening. In order to assess the preparedness of the ANC clinic to provide the services we used an index of seven indicators for 'clinic readiness' as follows:

- Availability of infrastructure Score $(0-5)$ : ANC waiting area, private space for ANC examination, and source of clean water, electricity, and laboratory.

- Availability of essential equipment: Score $(0-8)$ : blood pressure gauge, fetoscope, stethoscope, adult scale, measuring tape, Thermometer, Vaginal speculum (L, M, S), examination couch.

- Availability of guidelines, standards and registers (Score 0 - 8): ANC register, standard guidelines, protocol for focused ANC, revised ANC cards, Guidelines for PMTCT, Guidelines for diagnosis, treatment and prevention of malaria in pregnancy, Focused ANC job aid, malaria in pregnancy job aid, individual birth plan pamphlets.

- Availability of ANC essential drugs (Score 0 - 8): Iron folic, ferrous sulphate, Tetanus Toxoid, Sulfadoxine Pyrimethamine (SP), Amoxycillin, benzathine penicillin/PPF, erythromycin, metroniadazole (Flagyl).

- Availability of essential drugs for treatment of TB (Score 0 - 5): RHZE, EH, RHZ, HR, HRE. 
- Availability of essential drugs for PMTCT/ART (Score 0 - 5): Cotrimoxazole, Nevirapine, Zidovidine (ZDV, AXT) AZT syrup, Zidovidine +Lamividine (Combivir).

- Availability of laboratory supplies (Score 0-12): Reagents for urine test for glucose and protein, VDRL/RPR for syphilis, UNIGOLD, DETERMINE, BIOLINE (for HIV-rapid tests), Reagents for anemia tests (PCV), Reagents for TB tests, pregnancy test, Disposable gloves, urine specimen bottles, specimen pots for sputum; Disposable needles and syringes.

The index of indicators listed in Table 4 shows the mean score of "readiness" to provide the integrated ANC services before the intervention. Data was drawn from the clinic assessment tool and mean scores computed for each indicator, and then aggregated across all indicators to give the composite score for clinic preparedness, and compared across intervention clinics.

Table 4: Baseline facility "readiness" assessment

\begin{tabular}{l|cccccc}
\hline \multirow{2}{*}{ Availability of: } & $\begin{array}{c}\text { PGH } \\
\text { Kakamega }\end{array}$ & $\begin{array}{c}\text { St Mary's } \\
\text { Hospital }\end{array}$ & $\begin{array}{c}\text { Busia } \\
\text { DH }\end{array}$ & $\begin{array}{c}\text { Teso } \\
\text { DH }\end{array}$ & $\begin{array}{c}\text { Bungoma } \\
\text { DH }\end{array}$ & $\begin{array}{c}\text { Webuye } \\
\text { SDH }\end{array}$ \\
\hline Infrastructure (Score 0-5) & 5 & 5 & 5 & 4 & 5 & 5 \\
ANC equipment (Score 0-8) & 8 & 6 & 6 & 5 & 8 & 8 \\
Guidelines and materials (0-8) & 8 & 7 & 6 & 7 & 7 & 7 \\
ANC drugs (Score 0-8) & 8 & 8 & 8 & 7 & 8 & 2 \\
TB drugs (Score 0-5) & 5 & 5 & 5 & 4 & 5 & 4 \\
Drugs for PMTCT (Score 0-5) & 4 & 3 & 5 & 4 & 4 & 4 \\
Lab supplies (Score 0-12) & 9 & 12 & 9 & 10 & 8 & 12 \\
Total score (0 - 51) & 47 & 46 & 44 & 41 & 45 & 42 \\
\hline
\end{tabular}

Most of the hospitals in Western Province had the necessary equipment, supplies and infrastructure required for "readiness" (mean of $87 \%$, range 80 to $92 \%$ ). This assessment data demonstrates that facilities were ready to be able to integrate TB screening into FANC. Given the high level of facility readiness this assessment was not repeated at the endline.

\section{Provider knowledge of focused $A N C$ and $T B$}

Prior to the intervention few health providers were orientated in the following national guidelines; PMTCT (56\%), TB guidelines (38\% and FANC (27\%). Nevertheless $67 \%$ of all providers knew that pregnant women should attend ANC at least four times.

Health providers were asked about the key elements of FANC. An aggregate score includes knowledge on prevention of malaria, anemia in pregnancy, potential danger signs in pregnancy, counseling on PMTCT and individual birth planning, management of syphilis and screening for TB. The following scores were developed for provider knowledge indicators on:

- $\quad$ Prevention of malaria (Counsel client about use of treated bed nets, Intermittent preventive treatment (IPT), Case management of symptomatic women)

- Anemia in pregnancy (Hemoglobin level, examine client's palms, nailbeds, inner eyelids and tongue, give ferrous sulphate $\&$ folic acid, treat hookworms, give IPT, Counsel client about eating a balanced diet) 
- Management of syphilis (All pregnant women should be tested for syphilis, if positive, she and her partner should be treated with benzathine)

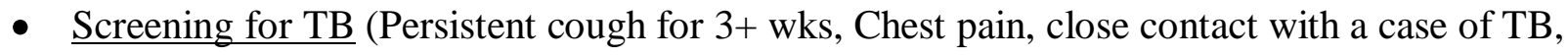
loss of body weight, intermittent fever and night sweats)

- Danger signs in pregnancy (Any vaginal bleeding, severe headache or blurred vision, swelling in the face, hands, legs, high fever, labored breathing, premature labor pains, baby moving less or not moving at all)

- Counseling on PMTCT (Obtain VCT to determine whether or not she is HIV+, STI by using condoms and having faithful partner, Avoid adding HIV virus to mother's system by practicing safe sex, Attend ANC clinic, Obtain ARV drugs for mother and infant around birth)

- Individual birth planning (When baby is due for delivery, identifying a skilled birth attendant, Identify a health facility for any emergency, Identifying a health facility for delivery, Identifying a decision-maker in case of emergency, putting money aside in case of emergency, planning transport in case of emergency and delivery, having a birth partner, collecting and preparing the basic supplies for birth)

Table 5 demonstrates the mean knowledge scores of the providers working in TB unit, PMTCT/ANC and the laboratory. Scores were highest among providers in ANC/PMTCT and lowest among laboratory technicians. The team training ensured use of different skills of the providers to ensure linkages and referral of pregnant women between the ANC, TB unit and laboratory.

Table 5: Mean knowledge scores at baseline by provider type (2005)

\begin{tabular}{l|cccccccc}
\hline \multirow{3}{*}{ Indicator } & \multicolumn{9}{|c}{ Provider working in: } \\
\cline { 2 - 9 } & \multicolumn{4}{|c}{ ANC clinic } & TB unit & \multicolumn{4}{c}{ Lab } & Total \\
\cline { 2 - 9 } Prevention of malaria in pregnancy (0-3) & Mean & $\mathrm{N}$ & Mean & $\mathrm{N}$ & Mean & $\mathrm{N}$ & Mean & $\mathrm{N}$ \\
Detection/ prevention of anemia (0-6) & 2.5 & 23 & 2.2 & 9 & 1.0 & 2 & 2.4 & 34 \\
Management of syphilis (0-2) & 5.0 & 23 & 4.9 & 9 & 2.5 & 2 & 4.8 & 34 \\
Screening for TB (0-5) & 1.6 & 22 & 1.9 & 9 & 1.5 & 4 & 1.7 & 35 \\
Danger signs in pregnancy (0-7) & 3.0 & 21 & 4.6 & 9 & 0 & 2 & 3.2 & 32 \\
Counseling on PMTCT (0-5) & 3.8 & 23 & 3.4 & 9 & 1.0 & 2 & 3.6 & 34 \\
Counseling on birth planning (0-9) & 2.3 & 23 & 3.2 & 5 & 1.0 & 2 & 2.3 & 30 \\
\hline
\end{tabular}

Antenatal providers had higher knowledge of the prevention or detection of malaria, syphilis and anemia. The aggregate score for ANC was 4.47 out of 7 (64\%). Although the PMTCT/ANC providers $(n=23)$ scored highest overall, the TB clinic providers $(n=9)$ scored well on knowledge of management of syphilis (94\%) and as might be expected screening for TB (91\%). The highest score among laboratory staff $(n=13)$ was for management of syphilis at $23 \%$.

Provider knowledge on signs of TB $(n=32)$ were encouraging; over $87 \%$ could recall a persistent cough was a key sign; as well as contact with person with TB $(81 \%)$, night sweats and intermittent fever (53\%), weight loss (53\%) and chest pain (47\%). Over half of the providers (58\%) said they would refer their client with these signs to the TB/chest clinic. 


\section{Quality of care}

Detection of existing diseases during provider-client observations at baseline (2005)

Providers refer clients for urine test (for protein, sugar and acetone) during most consultations (93\%). However the frequency of referrals for urine test decreased with subsequent ANC visits.

Providers were observed referring clients for syphilis and anemia test during the first visit only. Mostly, clients are significantly more likely to be referred for urine test and HIV test during the first visit but the frequency of referral greatly diminishes with subsequent ANC visits. None of the providers referred clients for TB tests through the four visits at baseline. Facilities did not link the ANC clinic to the TB clinics before the intervention (Table 6).

Table 6: Proportion of client - provider interactions in which clients were referred for tests by visit at baseline

\begin{tabular}{l|cccc}
\hline \multirow{3}{*}{ Procedures referred } & $A N C$ & $A N C$ & $A N C$ & $A N C$ \\
& Visit $_{1}$ & Visit $_{2}$ & Visit $_{3}$ & Visit $_{4}$ \\
\cline { 2 - 6 } & $\mathrm{n}=69$ & $\mathrm{n}=56$ & $\mathrm{n}=44$ & $\mathrm{n}=52$ \\
Refer client for urine test & $93 * *$ & 11 & 9 & $\%$ \\
Refer for a syphilis test & 91 & 0 & 0 & 0 \\
Refer for anemia test & 91 & 0 & 0 & 6 \\
Refer for HIV test & $90 *$ & 23 & 11 & 11 \\
\hline Refer for TB test & 0 & 0 & 0 & 0 \\
\hline \multirow{2}{*}{$* p<0.05 * * p<0.01$ (comparison between Visit 1 and 2 only) }
\end{tabular}

\section{Limited laboratory investigations at baseline}

Prior to the intervention, none of the clients had a TB test in any of the visits observed, Table 7 demonstrates that clients were more frequently referred for urine, HIV and Hb tests, and this was more likely to occur during the first ANC visit.

Table 7: Proportion of clients who received laboratory tests by visit at baseline

\begin{tabular}{l|cccc}
\hline \multirow{3}{*}{ Procedures performed } & $A N C$ & ANC & ANC & ANC \\
& Visit $_{1}$ & Visit $_{2}$ & Visit $_{3}$ & Visit $_{4}$ \\
\cline { 2 - 5 } & $\mathrm{n}=69$ & $\mathrm{n}=56$ & $\mathrm{n}=44$ & $\mathrm{n}=52$ \\
Urine tests & $\%$ & $\%$ & $\%$ & $\%$ \\
HIV tests & $70 * *$ & 9 & 7 & 2 \\
Blood test for HB & 79 & 16 & 14 & 6 \\
Syphilis test (visit 1 only) & 70 & 0 & 0 & 2 \\
TB test & 7 & 0 & 0 & 0 \\
\hline
\end{tabular}

$* * p<0.01$ (comparison between visit 1 and visit 2 ) 


\section{Screening for TB}

Table 8 indicates the proportion of client-provider interactions where providers asked about TB signs and symptoms during the ANC consultations. Significant increases were noted for nearly all screening questions at all four visits. The largest increases were noted for the first visit. The mean scores (out of five) improved significantly in all four visits. Nevertheless many providers are still failing to ask all five screening questions all the time. Even at the first visit only about half asked these five questions, and this deteriorated to about one-fourth of the questions in later visits. Nevertheless, this represents a significant improvement in screening overall.

Table 8: Proportion of clients who received screening of TB during ANC visits at Baseline and Endline

\begin{tabular}{|c|c|c|c|c|c|c|c|c|}
\hline \multirow{2}{*}{$\begin{array}{l}\text { Provider } \\
\text { observed asking } \\
\text { about: }\end{array}$} & \multicolumn{2}{|c|}{$A N C$ Visit 1} & \multicolumn{2}{|c|}{$A N C$ Visit 2} & \multicolumn{2}{|c|}{$A N C$ Visit 3} & \multicolumn{2}{|c|}{$A N C$ Visit 4} \\
\hline & $\begin{array}{c}\text { Base } \\
\mathbf{n}=69 \\
\%\end{array}$ & $\begin{array}{c}\text { End } \\
\mathrm{n}=36 \\
\%\end{array}$ & $\begin{array}{c}\text { Base } \\
\mathrm{n}=56 \\
\%\end{array}$ & $\begin{array}{c}\text { End } \\
n=36 \%\end{array}$ & $\begin{array}{c}\text { Base } \\
\mathrm{n}=44 \\
\%\end{array}$ & $\begin{array}{c}\text { End } \\
n=36 \%\end{array}$ & $\begin{array}{c}\text { Base } \\
\mathrm{n}=\mathbf{5 2} \\
\%\end{array}$ & $\begin{array}{c}\text { End } \\
\mathbf{n}=25 \\
\%\end{array}$ \\
\hline $\begin{array}{l}\text { Persistent cough } \\
\text { for } 3+\text { weeks }\end{array}$ & 3 & $67 * *$ & 0 & $47 * *$ & 2 & $33 * *$ & 4 & $36 * *$ \\
\hline Chest pain & 9 & $44 * *$ & 4 & $33 * *$ & 5 & $22 *$ & 6 & $28 * *$ \\
\hline $\begin{array}{l}\text { Close contact with } \\
\text { TB }\end{array}$ & 58 & 58 & 9 & $42 * *$ & 5 & $19 *$ & 4 & $28 * *$ \\
\hline $\begin{array}{l}\text { Loss of body } \\
\text { weight }\end{array}$ & 0 & $39 * *$ & 0 & $25 * *$ & 0 & $22 * *$ & 0 & $24 * *$ \\
\hline $\begin{array}{l}\text { Intermittent fever } \\
\text { and night sweats }\end{array}$ & 0 & $44 * *$ & 0 & $28 * *$ & 0 & $20 * *$ & 0 & $24 * *$ \\
\hline $\begin{array}{l}\text { Mean score } \\
(0-5)\end{array}$ & .7 & $2.53 * *$ & .13 & $1.75 * *$ & .11 & $1.17 * *$ & .13 & $1.40 * *$ \\
\hline
\end{tabular}

No referrals or tests for TB were observed before the intervention. At endline, providers were referring about five percent of clients for TB tests during the first three visits. All providers who referred clients for a TB test also advised them to return the following day to provide additional specimens.

\section{Scaling up activities}

In early 2008 the $\mathrm{MOH}$ replicated the introduction of screening for TB in ANC in a poor urban environment. Pumwani Maternity Hospital is one of the largest maternities in sub-Saharan Africa, with many of its clients living in informal crowded settlements in Nairobi.

The readiness score for Pumwani (38 out of 51, or about $75 \%$ ) was less than that of the Western Province hospitals, mainly because there is no TB unit and therefore no TB tests, drugs or guidelines. Provider knowledge was similar to that of the hospitals in Western Province, although the Pumwani providers had less knowledge of TB. All clients who are suspected to 
have TB are referred outside of the facility. Plans include making the TB test and drugs available on site so that pregnant women with $\mathrm{TB}$ can receive comprehensive care in one place.

Over one hundred service providers from Pumwani Maternity Hospital (78) and from private and public feeder clinics (31) were oriented in TB /FANC in five separate training sessions between February and April 2008. Facilitators included trainers from Population Council, the Pumwani Midwifery School and the Provincial Nursing Office. An evaluation of the impact of the training and supervision will be carried out later in the year through a separate funding mechanism.

A baseline assessment was also carried out in early 2008 in the remaining district hospitals in Western Province. This information is being made available for APHIA II Western which will take the lead in strengthening screening for TB in ANC in those facilities and across the province.

\section{DISCUSSION AND CONCLUSIONS}

The evidence from Western Province suggests that introducing screening for TB into ANC is both feasible and acceptable. The high level support from NLTP and DRH and the rapid incorporation of TB module into the National ANC orientation package demonstrates the commitment to intensify TB detection and provision of comprehensive care for women during pregnancy.

However the integration of TB screening into FANC was only tested out in hospital maternal and child health clinics, and where laboratories and the necessary equipment, drugs and supplies are more likely to be available. In addition the success of the integration is built on the knowledge level and commitment of the health providers at all levels of the health system.

Although this approach appears quite feasible there are some changes needed in operational policies for successful scale-up. One key element was to bring providers from the ANC, TB unit and laboratory together from each facility for team training as one unit. This provided a foundation to improve referrals and a consistent approach to meeting the needs of pregnant women who have TB.

Districts that wish to increase screening for TB at lower level facilities such as health centers and dispensaries must review the referral mechanisms for ensuring that pregnant women suspected of having TB do not have to travel far. The provision of sputum pots at community level that could then be transported by public health technicians (PHTs) on certain days would reduce the burden for the pregnant women. The acceptability of TB screening in ANC is demonstrated through the widespread use of the new orientation package as well as ease in which the providers have taken it on board within the FANC services. Screening for TB has become part of an everyday activity for the majority of nurses.

Effectiveness of integrating TB into ANC is demonstrated in the number of pregnant women were detected to have TB and were started on treatment. Although it is estimated that half of all those with HIV are also infected with $\mathrm{TB}$, the women attending antenatal care appear to be a 
reasonably 'healthy' population. Nevertheless, the simplicity of this approach means that women can be screened for TB in the antenatal setting as well as replicated in postnatal or other RH environments. It is not known what the risks of progression to TB in the postpartum period might be. Screening for TB may also be a more effective intervention in high TB and HIV prevalence areas.

In developing the tools for the study (TB module and job aids), program leaders from the NLTP and DRH were brought together, probably for the first time, to address the needs of women who may have TB. For the NLTP every case of TB detected is of value given their strategic objective in increasing case detection.

Recommendations for key stakeholders emerging from this effort include:

- Hold further consultations with key actors to ensure institutionalization and standardization of TB screening in ANC. This includes the integration or linkages with PMTCT- HIV and STI and postnatal services, and incorporating or updating curricula of pre-service training institutions and professional bodies on TB case management guidelines.

- Scale up TB screening in other service areas such as postnatal care through the training of providers with a focus on providing a comprehensive package of care for the mother and her infant. In addition, scale up screening of TB should be extended to the provinces with the highest TB rates. 


\section{REFERENCES}

Africa Science News Service, Fears of Resistant TB Strain in Kenya, Updated April 1, 2008.

Arora V.K et al, Tuberculosis and Pregnancy, Indian Journal of Tuberculosis, 2003, 5013

AVERT, Preventing Mother-to-Child Transmission Worldwide, Last updated April 1, 2008.

Khan M, Pillay T, Moodley JM, Connolly CA; Maternal mortality associated with tuberculosisHIV-1 co-infection in Durban South Africa. AIDS, 2001 September, 28:15(14):1857-63.

Pillay T, Khan M, Moodley J, Adhikari M, and Coovadia H. "Perinatal Tuberculosis and HIV-1: considerations for resource-limited settings", Lancet Infect Dis. 2004 Mar; 4(3): 155-165.

USAID Bureau for Africa, Office of Sustainable Development Tuberculosis in Africa Old Scourge, New Alliance, No. 1. 2000.

WHO (Communicable Disease) Global Tuberculosis Control, Surveillance, Planning, and Financing, WHO Report, (Geneva) 2003 and 2006. 
Appendix 1: TB job aid

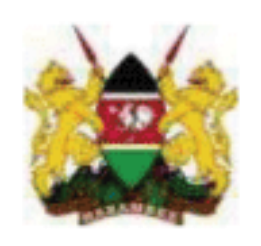

\section{Screening for TB in pregnancy}

Ask every pregnant woman at every

$A N C$ visit the following questions:

1. Have you had a persistent cough for more than two weeks?

2. Have you experienced excessive sweating or fever at night?

3. Have you lost any weight?

4. Do you have any chest pain?

5. Have you been in contact with anyone who has TB?

6. Do you have any swollen glands?

(This response can be confirmed during the physical examination of the pregnant woman) 
